Pacific Journal of Mathematics

PLANAR SURFACES IN KNOT MANIFOLDS 


\title{
PLANAR SURFACES IN KNOT MANIFOLDS
}

\author{
HOWARD LAMBERT
}

Let $K$ be a knot manifold, that is the 3-sphere $S^{3}$ minus an open regular neighborhood of a polygonal simple closed curve in $S^{3}$. Whether $K$ can be embedded in $S^{3}$ differently or in a homotopy 3-sphere different from $S^{3}$ (if such really exist) leads in a natural way to the question of which planar surfaces can be embedded in $K$. Geometric conditions are imposed on the embedded planar surfaces which are sufficient to imply that $K$ is not knotted, that is $K$ is homeomorphic to a disk cross $S^{1}$.

1. Introduction and definitions. In this paper we consider some geometric problems motivated by the so called "Property $P$ " [3] of a knot manifold $K$. In particular, we will investigate whether there is a continuous map $f$ of a planar surface $S$ (compact, submanifold of $E^{2}$ ) into $K$ such that $f(\operatorname{Int} S) \subset \operatorname{Int} K, f \mid B d S \subset B d K$ and $f$ is 1-1 on each component $\Delta_{1}, \cdots, \Delta_{n}$ of $B d S$ (each $\Delta_{i}$ is a simple closed curve (scc)). We are interested in the cases of either $I$. $f$ is $1-1$ and no $f\left(\Delta_{i}\right)$ is contained in a disk on $B d K$ or $I I . S$ is connected, $f\left(\Delta_{1}\right)$ is parallel to $K^{\prime} s$ longitude and each $f\left(\Delta_{i}\right), 2 \leqq i \leqq n$, is parallel to a fixed exotic homotopy killer of $K$ (definitions below). For example, if ${ }_{{ }_{1}}(K) \neq Z$, Case II holds and the homotopy killer of $K$ is exotic, then we would have a counter-example to "Property $P$ ". Conversely, if we had a $K$ violating "Property $P$ ", then there exists $f: S \rightarrow K$ as in Case II and each $f\left(\Delta_{i}\right), 2 \leqq i \leqq n$, is parallel to an exotic homotopy killer of $K$. In Theorem 1 we develop a geometric condition which is sufficient to imply $K$ is unknotted and in Theorem 2 we develop a related geometric condition which is sufficient to imply $K$ has "Property $P$ ".

Everything here is taken to be polyhedral. Definitions for such terminology as "properly embedded" and "boundary-irreducible" may be found in [17]. A knot manifold $K$ is a submanifold of $S^{3}$ such that $C l\left(S^{3}-K\right)$ is a solid torus $T=S^{1} \times D^{2}$. On $B d K$, but not separating $B d K$, there exists a unique (up to isotopy on $B d K$ ) sec homologous to zero (Mod $Z$ ) in $K$, called $K$ 's longitude. A meridian of $K$ is $x \times B d D^{2}, x \in S^{1}$, and we call it $K^{\prime} s$ ordinary homotopy killer. Any other sec on $B d K$ which kills $\pi_{1}(K)$ (by attaching a 3-cell along this scc) will be called an exotic homotopy killer. An exotic homotopy killer is of the form $m(l)^{n}$, where $m$ is the meridian of $K, l$ is the longitude of $K$ and $n \neq 0$. If $K$ has no exotic homotopy killer, then $K$ is said to have "Property $P$ ". Some results on "Property $P$ " have been obtained by R. Bing and J. Martin [3], A. C. Connor [4], F. 
Gonzales [9], J. Hempel [12] and J. Simon [15]. Results about the existence of surfaces (singular or not) in 3-manifolds have been obtained by W. R. Alford [2], C. Feustel [5], C. Feustel and N. Max [6], W. Heil [11], J. Hempel and W. Jaco [13], H. Lambert [14], J. Simon [16], and F. Waldhausen [18] among others.

2. Results for Case I. Suppose $f: S \rightarrow K$ as in Case I (since $f$ is a homeomorphism, identify $S$ with $f(S)$ ) and that each $\Delta_{i}$ is not parallel to $K^{\prime} s$ ordinary homotopy killer. Let $X_{n}$ be the 3-manifold obtained by adding $T\left(=\mathrm{Cl}\left(S^{3}-K\right)\right)$ to a regular neighborhood, $S \times[0,1]$, of $S$ in $K$ (see Figure 1 for a picture of an $X_{3}$ with $S$ connected).

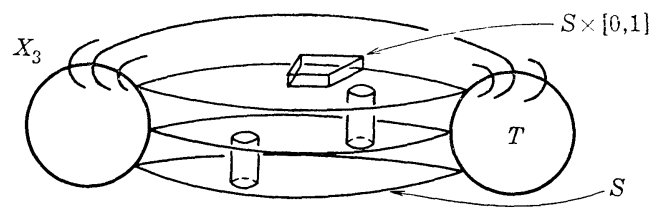

FIGURE 1

Recall from the first paragraph that $n$ is the number of boundary components of $S$ and picture $X_{n}$ as being obtained by attaching $B d S \times[0,1]$ to $n$ disjoint annuli $A_{1}, \cdots, A_{n}$ on $B d T$.

\section{LEMma 1. $X_{n}$ is boundary-irreducible.}

Proof. Assume $S$ is connected, as the proof is similar if not. Suppose $B d X_{n}$ is compressible, i.e., there exists a properly embedded disk $D$ in $X_{n}\left(B d D \subset B d X_{n}\right.$ and Int $\left.D \subset \operatorname{Int} X_{n}\right)$ such that $B d D$ does not bound a disk in $B d X_{n}$. Put $D$ in general position relative to $\bigcup_{i=1}^{n} A_{i}$. After removing simple closed curves of $D \cap \bigcup_{i=1}^{n} A_{i}$ which bound disks in $\bigcup_{i=1}^{n} A_{i}$, it follows that there exists a subdisk $D^{\prime}$ of $D$ such that either 1. $D^{\prime}=D$ and $D^{\prime} \cap\left(\bigcup_{i=1}^{n} A_{i}\right)=\varnothing, 2 . B d D^{\prime} \subset A_{i}$ and Int $D^{\prime} \cap\left(\bigcup_{i=1}^{n} A_{i}\right)=$ $\varnothing$ or 3. $B d D^{\prime}$ consists of two arcs, one in $B d X_{n}$ and the other in $A_{i}$, and Int $D^{\prime} \cap\left(\bigcup_{i=1}^{n} A_{i}\right)=\varnothing$. In Case 1 , if $D \subset S \times[0,1]$, then it follows by Proposition 3.1 of [17] that $B d D$ bounds a disk in $B d X_{n}$, contradiction. If $D \subset T$, then either each $f\left(\Delta_{i}\right)$ is parallel to $K^{\prime} s$ ordinary homotopy killer, contradiction, or $B d D$ bounds a disk in $B d X_{n}$, contradiction. Case 2 cannot occur since the center line of each $A_{i}$ is not homologous to zero in either $S \times[0,1]$ or $T$. In Case 3 if $D^{\prime} \subset S \times[0,1]$, the arc $B d D^{\prime} \cap A_{i}$ intersects one boundary component of $A_{i}$ and, by using Proposition 3.1 of [17], the number of components of $D \cap\left(\bigcup_{i=1}^{n} A_{i}\right)$ can be reduced. Similarly, in Case 3 for $D^{\prime} \subset T$ it follows that the number of components of $D \cap\left(\bigcup_{i=1}^{n} A_{i}\right)$ can be reduced (assume $n>1$, since $X_{1}$ is a 3-cell). All three cases now imply $D$ could not have existed and 
therefore $X_{n}$ is boundary-irreducible.

Suppose $M$ is a 3-manifold. If $D$ is a disk properly embedded in $M$ such that $B d D$ does not bound a disk on $B d M$, then we say $M$ has a handle $D$. More generally, if $S$ is a connected planar 2-manifold properly embedded in $M$ such that $1 . n$, the number of boundary components $\Delta_{1}, \cdots, \Delta_{n}$ of $S$, is odd and 2 . there exists an annulus $A=$ $S^{1} \times[1, n]$ on $B d M$ such that each $\Delta_{i}=S^{1} \times \mathrm{i}, 1 \leqq i \leqq n$, then call $A$ handle-like in $M$.

LemMa 2. Suppose $M$ is a 3-manifold with a handle $D$ and $a$ handle-like annulus $A$. Then $M$ has a handle $D_{0}$ such that $D_{0} \cap A=\varnothing$ and $A$ is handle-like in $M-D_{0}$.

Proof. The case $n=1$ is easy. Suppose then that $n \geqq 3$ (and $n$ odd) but that $B d D \cap A=\varnothing$ (we may need to pull $B d D$ off $A$ by an isotopy in $B d M$ to achieve this). If $S$ is in general position relative to $D$, we may choose a subdisk $D^{\prime}$ of $D$ such that $B d D^{\prime} \subset S$ and Int $D^{\prime} \cap S=\varnothing$. Now cut $S$ at $B d D^{\prime}$ and fill in the resulting two holes by disks close to but on opposite sides of $D^{\prime}$ to obtain two planar surfaces, at least one of which, $S^{\prime}$, has an odd number of boundary components $\left(B d S^{\prime} \subset B d S\right)$ and $S^{\prime} \cap D$ has fewer components than $S \cap D$. Repeating this argument a finite number of times yields $D_{0}(=D)$ in this special case.

Now suppose $B d D \cap A(\neq \varnothing)$ consists of arcs, each connecting one boundary component of $A$ to its other, and that $D \cap S$ consists of ares only (simple closed curves may be removed as in the special case). Note that each arc of $D \cap S$ starts and ends in $B d D \cap A$ and that $n$ such arcs start at each arc of $B d D \cap A$. If an arc of $D \cap S$ starts and ends on the same arc of $B d D \cap A$, then there exists a subdisk $D^{\prime}$ of $D$ such that $D^{\prime} \cap A$ is an arc on $B d D^{\prime}$, the complementary are of $B d D^{\prime}$ is contained in $D \cap S$ and $\operatorname{Int} D^{\prime} \cap S=\varnothing$. Now cut $S$ at $B d D^{\prime} \cap S$ and attach two disks close to but on opposite sides of $D^{\prime}$. The resulting $S^{\prime}$ then contains one boundary component which bounds a disk in $A$. Fill in this boundary component to obtain $S^{\prime \prime}$ such that $S^{\prime \prime}$ is planar, $B d S^{\prime \prime} \subset B d S$ and $S^{\prime \prime}$ has $n-2$ boundary components.

If no arc of $D \cap S$ has both its end points in the same arc of $B d D \cap A$, then, in $D$, there are two adjacent $\operatorname{arcs} Q_{1}, Q_{2}$ of $B d D \cap A$ (relative to $B d D)$ such that $Q_{1} \times(n+1) / 2\left(=Q_{1} \cap \Delta(n+1) / 2\right)$ is connected to $Q_{2} \times(n+1) / 2$ by an arc $\gamma_{0}$ of $D \cap S$. Since $S$ is orientable and $\gamma_{0}$ has both ends in the same boundary component of $S$, namely $\Delta(n+1) / 2, \gamma_{0}$ does not separate $Q_{1} \times 1$ from $Q_{2} \times 1$ in $D$. Hence there is an arc of $D \cap S$ with both ends in $\Delta_{1}\left(\right.$ or $\left.\Delta_{n}\right)$. Since all arcs of $D \cap S$ with one end point in $\Delta_{1} \cup \Delta_{n}$ have both end points in $\Delta_{1} \cup \Delta_{n}$, we may ignore all these arcs and repeat the above argument $((n+1) / 2)-2$ 
times more to conclude that for each boundary component $\Delta_{i}$ of $S$ there exists an arc of $S \cap D$ with both endpoints in $\Delta_{i}$. Since $S$ is planar, one of these arcs together with an arc on $B d S$ bounds a disk $D^{\prime}$ in $S$ such that Int $D^{\prime} \cap D=\varnothing$. Now cut $D$ at $B d D^{\prime} \cap D$ and attach two disks close to but on opposite sides of $D^{\prime}$ to obtain two disks properly embedded in $M$ and at least one of them is a handle of $M$ which intersects $A$ in fewer arcs than $D$ does. Applying the various cases above a finite number of times yields the desired handle $D_{0}$.

It follows as a corollary to Lemma 2 that if $M$ is a cube with handles, then $n=1$, i.e., the center line of $A$ bounds a disk in $M$.

THEOREM 1. Suppose $f: S \rightarrow K$ as in Case I, that $f(S)$ has at least two components $S_{1}, S_{2}$ such that each has an odd number of boundary components and that there exists an annulus on BdK whose boundary separates $B d S_{1}$, from $B d S_{2}$ in $B d K\left(=S^{1} \times S^{1}\right)$. Then $K$ is unknotted (homeomorphic to $T=\mathrm{Cl}\left(S^{3}-K\right)$ ).

Proof. Since $S_{1}$ and $S_{2}$ have an odd number of boundary components and no boundary component of $f(S)$ is contained in a disk on $B d K$, it follows that each boundary component of $f(S)$ is parallel to $K^{\prime} s$ longitude. Let $A_{1}, A_{2}$ be disjoint annuli in $B d K$, parallel to $K^{\prime} s$ longitude, such that $B d S_{1} \subset A_{1}$ and $B d S_{2} \subset A_{2}$. Let $U_{1}, U_{2}$ be disjoint regular neighborhoods of $S_{1} \cup A_{1}, S_{2} \cup A_{2}$ in $K$, respectively. Then $U_{1}$ is homeomorphic to an $X_{n}$ of Lemma 1; hence it is boundary-irreducible. Similarly $U_{2}$ is boundary-irreducible and by [7] it follows that there is a properly embedded disk $D$ in $\mathrm{Cl}\left(S^{3}-U_{1} \cup U_{2}\right)$ such that $B d D$ does not bound a disk in $B d\left(\mathrm{Cl}\left(S^{3}-U_{1} \cup U_{2}\right)\right)=B d U_{1} \cup B d U_{2}$. Suppose, without loss of generality, that $B d D \subset B d U_{1}$. Since $D \cap U_{2}=\varnothing$, it follows that we may cut $D$ and fill in on the two annuli components of $\mathrm{Cl}\left(B d K-U_{1} \cup U_{2}\right)$ so as to assume $D \cap T=\varnothing$ (note that obtaining $D \cap T=\varnothing$ involves assuming $K$ is knotted). Now add to $U_{1}$ a regular neighborhood of $D$ in $\mathrm{Cl}\left(S^{3}-U_{1} \cup U_{2}\right)$ to obtain a new 3-manifold $U_{1}^{\prime}$ (if $B d D$ separates $B d U_{1}$ also add the component of $\mathrm{Cl}\left(S-U_{1}\right)-D$ not containing $U_{2}$ to $\left.U_{1}^{\prime}\right)$. Note that the genus of $B d U_{1}^{\prime}$ is less than the genus of $B d U_{1}$. Repeat these steps on $U_{1}^{\prime}, U_{2}$. But now it is possible that $U_{1}^{\prime}$ is not boundary-irreducible. If $D \subset U_{1}^{\prime}$, Lemma 2 says we may assume $D \cap S_{1}=$ $\varnothing$ and cut out an open regular neighborhood of $D$ in $U_{1}^{\prime}$ to obtain the new $U_{1}^{\prime \prime}$. Again the genus of $B d U_{1}^{\prime \prime}$ is less than the genus of $B d U_{1}^{\prime}$. Continuing, we eventually conclude that there is a 3-cell $B$ in $K$ such that $B \cap B d K$ is either $A_{1}$ or $A_{2}$ and hence $K$ is unknotted.

3. Results for Case II. Suppose $f: S \rightarrow K$ as in Case II and, in addition, assume each $f\left(\Delta_{i}\right), 2 \leqq i \leqq n$, is parallel to a fixed exotic homotopy killer of $K$. We may also assume that $f$ is in general posi- 
tion, that is the singularities of $f$ on $S$ consist of pairwise disjoint arcs with endpoints in $B d S$ and $f$ sews these arcs together in pairs, each pair forming a single arc in the image (see W. Haken's [10] to see how to eliminate branch points and triple points at the expense of increasing $n$ ). There are two types of such arcs of singularities, Type $\alpha$ where the arc runs from $\Delta_{1}$ to some $\Delta_{i}, i \neq 1$, and Type $\beta$ where the arc has both endpoints in $\Delta_{1}$ and its associated arc runs from $\Delta_{i}$ to $\Delta_{j}$, $i, j>1$ and $i \neq j$. In [10], Haken shows that we can always make every arc of Type $\alpha$. Unfortunately, from the point of view of studying "Property $P$ ", Type $\alpha$ arcs seem to be particularly intractible. If all arcs are of Type $\beta$, then $K$ corresponds to being like a ribbon knot [8, p. 172] relative to its exotic homotopy killer. It is a very particular case of Type $\beta$ arcs we wish to look at. Suppose $S$ contains a pair of arcs $\beta_{1}, \beta_{2}$ of Type $\beta$ sewed together by $f$ where $B d \beta_{1} \subset \Delta_{1}$ and one of the two components of $S-\beta_{1}$ contains no other arc of singularity but $\beta_{2}$. Denote the closure of this component of $S-\beta_{1}$ by $\Gamma$ ( $\Gamma$ is a disk with 2 holes, see Figure 2 for a picture of $f(\Gamma) \cup T$ ).

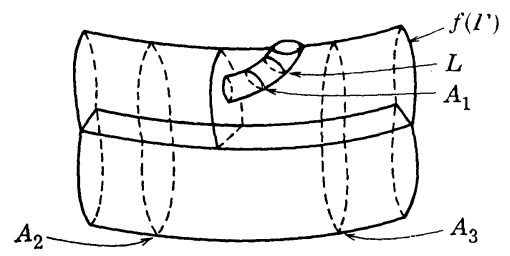

FIGURE 2

Theorem 2. Suppose 1. $f: S \rightarrow K$ as in Case II, 2. $S$ contains two (disjoint) $\Gamma$ 's, $\Gamma_{1}$ and $\Gamma_{2}$, and 3. n, the number of boundary components of $S$, is minimal with respect to property 1 . Then $K$ is unknotted.

Proof. First assume $n>1$, since $n=1$ implies, by Dehn's Lemma, that $K$ is unknotted. Let $A_{1}, A_{2}$ be disjoint annuli on $B d K$ such that $f\left(\Gamma_{1}\right) \cap B d K \subset A_{1}$ and $f\left(\Gamma_{2}\right) \cap B d K \subset A_{2}$. Let $U_{1}, U_{2}$ be disjoint regular neighborhoods of $A_{1} \cup f\left(\Gamma_{1}\right), A_{2} \cup f\left(\Gamma_{2}\right)$ in $K$, respectively. We claim both $U_{1}$ and $U_{2}$ are homeomorphic to an $X_{3}$ of Lemma 1. (To see this we have indicated in Figure 2 where the three annuli $A_{1}, A_{2}$ and $A_{3}$ of Lemma 1 would be located in $U_{1}$.) By Lemma $1, U_{1}, U_{2}$ are boundary irreducible and we follow the technique used in the proof of Theorem 1 to conclude that there is a disk $D$ properly embedded in $\operatorname{Cl}\left(S^{3}-U_{1} \cup U_{2}\right)$ such that $B d D \subset U_{1}$ (or $U_{2}$ ) and $D \cap T=\varnothing$. As in the proof of Theorem 1 , we add a regular neighborhood of $D$ to $U_{1}$ to obtain $U_{1}^{\prime}$. Now $B d U_{1}^{\prime}$ is a torus, $S^{1} \times S^{1}$. By [1], the closure of one complementary domain of $S^{1} \times S^{1}$ in $S^{3}$ is a solid torus $T^{\prime}$. If $f\left(\Gamma_{1}\right) \subset T^{\prime \prime}$, then the sce 
$L$ of Figure 2 can be shrunk to a point in $T^{\prime \prime}$ since homology and homotopy are the same in $T^{\prime \prime}$. (To see that $L$ is homologous to zero Mod $Z$, note that $L$ bounds an orientable surface in $f\left(\Gamma_{1}\right) \cup A_{1}$.) Suppose $f\left(\Gamma_{2}\right) \subset T^{\prime \prime}$. Then $T^{\prime \prime}-\left(\operatorname{Int} T \cup \operatorname{Int} A_{1}\right)$ is a solid torus, $L \sim 0 \operatorname{Mod} Z$ in $f\left(\Gamma_{2}\right) \cup A_{2} \subset T^{\prime}-\left(\operatorname{Int} T \cup \operatorname{Int} A_{1}\right)$ and hence the $L$ of $f\left(\Gamma_{2}\right)$ can be shrunk to a point in $T^{\prime}$ - Int $T$. In either case, by using the singular disk that $L$ bounds, it follows that there is an $f^{\prime}: S^{\prime} \rightarrow K$ as in Case II with $n^{\prime}<n$, contradicting property 3 of the hypothesis. Then $n=$ 1 and $K$ is unknotted.

4. A question. Suppose $f: S \rightarrow K$ as in Case II, each $f\left(\Delta_{i}\right)$, $2 \leqq i \leqq n$, is parallel to a fixed exotic homotopy killer of $K$ and each arc of singularity in $S$ is of Type $\beta$. We can say in general that there exist disjoint $\Gamma_{1}, \Gamma_{2}$ in $S$ as before but now $\Gamma_{1}, \Gamma_{2}$ contain holes whose boundaries go parallel to the exotic homotopy killer under $f$. It does not seem likely that $K$ is knotted if $\Gamma_{1}, \Gamma_{2}$ exist, but the author could not show this. We conclude then with the following

Question. If $K$ does not have "Property $P$ " and all singularities of the resulting $f: S \rightarrow K$ are of Type $\beta$, then is $K$ unknotted?

\section{REFERENCES}

1. J. W. Alexander, On the subdivision of 3-space by a polyhedron, Proc. Nat. Acad. Sci. U. S. A., 10 (1924), 6-8.

2. W. R. Alford and C. B. Schaufele, In topology of Manitoids, (1970), 87-96, Markham, Chicago, Illinois.

3. R. H. Bing and J. M. Martin, Cubes with knotted holes, Trans. Amer. Math. Soc., 155 (1971), 217-231.

4. A. C. Connor, manuscript.

5. C. Feustel, A splitting theorem for closed orientable 3-manifolds, to appear.

6. C. Feustel and N. Max, On a problem of R. H. Fox, to appear.

7. R. H. Fox, On the embedding of polyhedra in 3-space, Ann. of Math., 49 (1948), $462-470$.

8. - Some problems in knot theory, Topology of 3-manifolds and related topics, Prentice-Hall, 1962.

9. F. Gonzales, Thesis, Princeton University, 1970.

10. W. Haken, On homotopy 3-spheres, Illinois J. Math., 10 (1966), 159-180.

11. W. Heil, On the existence of incompressible surfaces in certain 3-manifolds, Proc. Amer. Math. Soc., 23 (1969), 704-707.

12. J. Hempel, $A$ simply connected 3-manifold is $S^{3}$ if it is the sum of a solid torus and the complement of a torus knot, Proc. Amer. Math. Soc., 15 (1964), 154-158.

13. J. Hempel and W. Jaco, 3-manifolds which fiber over a surface, manuscript.

14. H. Lambert, A 1-linked link whose longitudes lie in the second commutator subgroup, Trans. Amer. Math. Soc., 147 (1970), 216-269.

15. J. Simon, Some classes of knots with property P, in Topology of Manifolds, pp. 195199, Markham, Chicago, Ill., 1970.

16. - On knots with non-trivial interpolating manifolds, Trans. Amer. Math. Soc., 160 (1971). 
17. F. Waldhausen, On irreducible 3-manifolds which are sufficiently large, Ann. of Math., (2) 87 (1968), 56-88.

18. - Gruppen mit zentrum und 3-dimensionale mannigfaltigkeiten, Topology, 6 (1967), 505-517.

Received September 30, 1970 and in revised form February 14, 1971. Research supported by NSF Grant GP-19295. A portion of this work was done while the author was at the Institute for Defense Analysis, Princeton, New Jersey.

UNIVERSITY OF IOWA 



\title{
PACIFIC JOURNAL OF MATHEMATICS
}

\author{
EDITORS
}

\author{
H. SAMELSON \\ Stanford University \\ Stanford, California 94305 \\ C. R. HOBBY \\ University of Washington \\ Seattle, Washington 98105
}

J. DugundJI

Department of Mathematics

University of Southern California

Los Angeles, California 90007

RICHARD ARENS

University of California

Los Angeles, California 90024

\section{ASSOCIATE EDITORS}
E. F. BeCKenBaCH
B. H. NeUMANN
F. WOLF
K. Yoshida

\section{SUPPORTING INSTITUTIONS}

\author{
UNIVERSITY OF BRITISH COLUMBIA \\ CALIFORNIA INSTITUTE OF TECHNOLOGY \\ UNIVERSITY OF CALIFORNIA \\ MONTANA STATE UNIVERSITY \\ UNIVERSITY OF NEVADA \\ NEW MEXICO STATE UNIVERSITY \\ OREGON STATE UNIVERSITY \\ UNIVERSITY OF OREGON \\ OSAKA UNIVERSITY
}

\author{
UNIVERSITY OF SOUTHERN CALIFORNIA \\ STANFORD UNIVERSITY \\ UNIVERSITY OF TOKYO \\ UNIVERSITY OF UTAH \\ WASHINGTON STATE UNIVERSITY \\ UNIVERSITY OF WASHINGTON \\ AMERICAN MATHEMATICAL SOCIETY \\ NAVAL WEAPONS CENTER
}

The Supporting Institutions listed above contribute to the cost of publication of this Journal, but they are not owners or publishers and have no responsibility for its content or policies.

Mathematical papers intended for publication in the Pacific Journal of Mathematics should be in typed form or offset-reproduced, (not dittoed), double spaced with large margins. Underline Greek letters in red, German in green, and script in blue. The first paragraph or two must be capable of being used separately as a synopsis of the entire paper. The editorial "we" must not be used in the synopsis, and items of the bibliography should not be cited there unless absolutely necessary, in which case they must be identified by author and Journal, rather than by item number. Manuscripts, in duplicate if possible, may be sent to any one of the four editors. Please classify according to the scheme of Math. Rev. Index to Vol. 39. All other communications to the editors should be addressed to the managing editor, Richard Arens, University of California, Los Angeles, California, 90024.

50 reprints are provided free for each article; additional copies may be obtained at cost in multiples of 50 .

The Pacific Journal of Mathematics is published monthly. Effective with Volume 16 the price per volume (3 numbers) is $\$ 8.00$; single issues, $\$ 3.00$. Special price for current issues to individual faculty members of supporting institutions and to individual members of the American Mathematical Society: $\$ 4.00$ per volume; single issues $\$ 1.50$. Back numbers are available.

Subscriptions, orders for back numbers, and changes of address should be sent to Pacific Journal of Mathematics, 103 Highland Boulevard, Berkeley, California, 94708.

PUBLISHED BY PACIFIC JOURNAL OF MATHEMATICS, A NON-PROFIT CORPORATION

Printed at Kokusai Bunken Insatsusha (International Academic Printing Co., Ltd.), 270, 3chome Totsuka-cho, Shinjuku-ku, Tokyo 160, Japan. 


\section{Pacific Journal of Mathematics}

\section{Vol. 39, No. $3 \quad$ July, 1971}

William O'Bannon Alltop, 5-designs in affine spaces ................... 547

B. G. Basmaji, Real-valued characters of metacyclic groups ................. 553

Miroslav Benda, On saturated reduced products....................... 557

J. T. Borrego, Haskell Cohen and Esmond Ernest Devun, Uniquely representable semigroups. II.......................................

George Lee Cain Jr. and Mohammed Zuhair Zaki Nashed, Fixed points and stability for a sum of two operators in locally convex spaces ....................

Donald Richard Chalice, Restrictions of Banach function spaces ...............

Eugene Frank Cornelius, Jr., A generalization of separable groups ..............

Joel L. Cunningham, Primes in products of rings ......................

Robert Alan Morris, On the Brauer group of $Z$.

593

603

615

David Earl Dobbs, Amitsur cohomology of algebraic number rings ...............

Charles F. Dunkl and Donald Edward Ramirez, Fourier-Stieltjes transforms and

weakly almost periodic functionals for compact groups ...................

Hicham Fakhoury, Structures uniformes faibles sur une classe de cônes et

d'ensembles convexes ......................................

Leslie R. Fletcher, A note on C $\theta \theta$-groups.

Humphrey Sek-Ching Fong and Louis Sucheston, On the ratio ergodic theorem for

semi-groups............................................

James Arthur Gerhard, Subdirectly irreducible idempotent semigroups ...........

Thomas Eric Hall, Orthodox semigroups.....................

Marcel Herzog, $C \theta \theta$-groups involving no Suzuki groups ..........

669

687

John Walter Hinrichsen, Concerning web-like continua ..........

691

Frank Norris Huggins, A generalization of a theorem of F. Riesz.

695

Carlos Johnson, Jr., On certain poset and semilattice homomorphisms

703

Alan Leslie Lambert, Strictly cyclic operator algebras ...........

717

Howard Wilson Lambert, Planar surfaces in knot manifolds . . .

727

Robert Allen McCoy, Groups of homeomorphisms of normed linear spaces ....... 735

T. S. Nanjundiah, Refinements of Wallis's estimate and their generalizations ...... 745

Roger David Nussbaum, A geometric approach to the fixed point index .......... 751

John Emanuel de Pillis, Convexity properties of a generalized numerical range .... 767

Donald C. Ramsey, Generating monomials for finite semigroups ....

783

William T. Reid, A disconjugacy criterion for higher order linear vector differential equations...

Roger Allen Wiegand, Modules over universal regular rings...

Kung-Wei Yang, Compact functors in categories of non-archimedean Banach

spaces.

R. Grant Woods, Correction to: "Co-absolutes of remainders of Stone-Čech compactifications".

Ronald Owen Fulp, Correction to: "Tensor and torsion products of

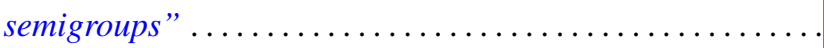

Bruce Alan Barnes, Correction to: "Banach algebras which are ideals in a banach 